\title{
Population analysis of open clusters: radii and mass segregation
}

\author{
E. Schilbach ${ }^{1}$, N. V. Kharchenko ${ }^{1,2,4}$, A. E. Piskunov ${ }^{1,3,4}$, S. Röser ${ }^{1}$, and R.-D. Scholz ${ }^{4}$ \\ 1 Astronomisches Rechen-Institut, Mönchhofstraße 12-14, 69120 Heidelberg, Germany \\ e-mail: [elena;nkhar; apiskunov; roeser] ari .uni-heidelberg.de \\ 2 Main Astronomical Observatory, 27 Academica Zabolotnogo Str., 03680 Kiev, Ukraine \\ e-mail: nkhar@mao.kiev.ua \\ 3 Institute of Astronomy of the Russian Acad. Sci., 48 Pyatnitskaya Str., Moscow 109017, Russia \\ e-mail: piskunov@inasan.rssi.ru \\ 4 Astrophysikalisches Institut Potsdam, An der Sternwarte 16, 14482 Potsdam, Germany \\ e-mail: [apiskunov;nkharchenko; rdscholz]@aip.de
}

Received 8 December 2005 / Accepted 23 May 2006

ABSTRACT

\begin{abstract}
Aims. Based on our well-determined sample of open clusters in the all-sky catalogue ASCC-2.5 we derive new linear sizes of some 600 clusters, and investigate the effect of mass segregation of stars in open clusters.

Methods. Using statistical methods, we study the distribution of linear sizes as a function of spatial position and cluster age. We also examine statistically the distribution of stars of different masses within clusters as a function of the cluster age.

Results. No significant dependence of the cluster size on location in the Galaxy is detected for younger clusters ( $<200 \mathrm{Myr})$, whereas older clusters inside the solar orbit turned out to be, on average, smaller than outside. Also, small old clusters are preferentially found close to the Galactic plane, whereas larger ones more frequently live farther away from the plane and at larger Galactocentric distances. For clusters with $\left(V-M_{V}\right)<10.5$, a clear dependence of the apparent radius on age has been detected: the cluster radii decrease by a factor of about 2 from an age of $10 \mathrm{Myr}$ to an age of $1 \mathrm{Gyr}$. A detailed analysis shows that this observed effect can be explained by mass segregation and does not necessarily reflect a real decrease of cluster radii. We found evidence for the latter for the majority of clusters older than $30 \mathrm{Myr}$. Among the youngest clusters (between 5 and $30 \mathrm{Myr}$ ), there are some clusters with a significant grade of mass segregation, whereas some others show no segregation at all. At a cluster age between 50 and 100 Myr, the distribution of stars of different masses becomes more regular over cluster area. In older clusters the evolution of the massive stars is the most prominent effect we observe.
\end{abstract}

Key words. stars: luminosity function, mass function - Galaxy: disk - Galaxy: evolution - open clusters and associations: general solar neighbourhood - Galaxy: stellar content

\section{Introduction}

Open star clusters are gravitationally bound systems of, typically, several hundreds of stars formed together. Primordial conditions during the cluster formation and the location of the parental molecular cloud in the Galaxy play an important role in the fate of a cluster. On the other hand, the stellar content of a cluster evolves with time, and internal and external interactions affect the properties of individual cluster members as well as of the whole cluster as a system. Therefore, the spatial structure and mass distribution that we observe today in a given cluster is the result of the original brand marks and the ongoing evolution.

Numerical simulations of the dynamical evolution predict a mass segregation in open clusters i.e., a different concentration of cluster members with different masses with respect to the cluster centre. This process occurs on approximately the relaxation time-scale and is independent of most of the possible initial conditions (Bonnell \& Davies 1998). During the dynamical evolution of a cluster, more massive members sink to the centre, whereas less massive stars tend to show a diffuse distribution (Portegies Zwart \& McMillan 2002). A relaxed cluster can be thought of as a set of nested spherical clouds of stars of different mass (e.g., see Adams et al. 2001, for illustration). With increasing mass of the stars, the radial density profile becomes steeper and narrower. Due to tidal interactions with the Galactic gravitational field, the cluster can lose stars once they overflow its tidal radius. Due to mass segregation a cluster loses preferentially low mass stars from the cluster corona, which evaporate into the field, up to an entire dissolution of the cluster in the Galaxy (Andersen \& Nordström 2000). A sudden mass loss or close passage of giant molecular clouds can considerably disturb a relaxation process and reduce the life-time of open clusters (Kroupa et al. 2001; Bergond et al. 2001).

Predicted by simulations (Spitzer \& Shull 1975), mass segregation was already found in many open clusters. The most reliable results on mass segregation can be expected for nearby clusters like the Pleiades and Praesepe, where cluster members can be observed over a wide range of magnitudes and masses. Compared to the distribution of more massive stars, indications for a flatter density profile of cluster members with $m<1 m_{\odot}$ were obtained in these clusters by Jones \& Stauffer (1991), and more recently by Adams et al. (2001, 2002) who used data from 2MASS and USNO-A. Raboud \& Mermilliod (1998a,b) found evidence for a continuous flattening of density profiles with decreasing mass of cluster members in the Pleiades and Praesepe, and in a much more distant open cluster, NGC 6231, too. Similar effects were detected by Sagar et al. (1988), who 
considered 11 distant clusters in the Galactic disk and by de Grijs et al. (2002a,b,c), who studied mass segregation in open clusters in the Large Magellanic Cloud.

Additionally to the mass segregation in older clusters due to dynamical evolution, a higher concentration of massive stars to the centre was also found in some very young clusters (e.g., in the Orion Nebula Cluster, Hillenbrand 1997; Hillenbrand \& Hartmann 1998). Due to the youth of these clusters, the central location of massive stars can not be explained by dynamical evolution only. Additional arguments from star formation scenarios and early cluster dynamics have to be considered (Bonnell \& Davies 1998; Kroupa et al. 2001).

Since mass segregation has a direct impact on the spatial structure of clusters, the effect should be seen in the apparent cluster sizes. In this context, the observed cluster size is an important parameter related to the dynamical state both of the cluster and of the Galactic disk. As open clusters are found over a broad span of ages, a study of global trends including cluster size, if they exist, should be possible from a representative sample of clusters with homogeneous data on the main cluster parameters.

Practically all famous collections (Trumpler, Collinder etc.) of cluster data include estimations of angular sizes of open clusters, but the first systematic determination of apparent diameters was made by Lyngå (1987) for about 1000 open clusters from visual inspection of the POSS prints. These estimates are included in the Lund catalogue, 5th edition, together with about other 150 clusters, where an estimate by G. Lyngå himself was not available, hence taken from the references quoted in the catalogue. For reasons of homogeneity only the diameter of the cluster nucleus (core) was included in the catalogue, although already then it was known that some clusters showed coronae. These data were used by Lyngå (1982) and Janes et al. (1988) for their studies of properties of the open cluster system. Since that time ages and distances were available for a small fraction of known clusters, their sample included about 400 clusters. No estimations were made on how well this sample represents the Galactic cluster population.

Although structural parameters have been derived for many individual clusters during the last decade, there are only a few studies dealing with a systematic determination of cluster sizes based on objective and uniform approaches for larger cluster samples. Danilov \& Seleznev (1993) derived structural parameters for 103 compact distant $(>1 \mathrm{kpc})$ clusters from star counts down to $B \approx 16$ from homogeneous wide-field observations with a $50-\mathrm{cm}$ Schmidt camera of the Ural university. Based on $U B V$-CCD observations compiled from literature, Tadross et al. (2002) redetermined ages and distances for 160 open clusters. The cluster sizes were estimated visually, from POSS prints, and they are practically identical to the diameters estimated by Lyngå. Kharchenko et al. (2003) determined radii of about 400 clusters from star counts in ASCC-2.5 and USNO-A2.0 catalogues. Nilakshi et al. (2002) derived structural parameters of 38 open clusters selected from the Lyngå's (1987) catalogue from star counts in the USNO-A2.0 catalogue. Recently, Bonatto \& Bica (2005) published structural and dynamical parameters of 11 open clusters obtained from star counts and photometric membership based on the 2MASS survey.

Correlations of cluster size with age and Galactic location were found by some of the authors above, though the results are rather controversial (see Sects. 3 and 4 for more details). There are at least two major aspects which must be taken into account in the interpretation of the results. At first, how well does a given sample represent the local population of open clusters in the Galaxy, or which biases can arise from the incompleteness of the data and influence the results. Second, how homogeneous are data on individual clusters, on their size, age, distance, provided that they are based on observations with different telescopes equipped with different detectors, or if different methods were used for the determination of cluster parameters. The answer is not trivial considering the large set of data compiled from literature, especially.

Using the Catalogues of Open Cluster Data $\left(\mathrm{COCD}^{1}\right.$; Kharchenko et al. 2004, 2005a,b, Papers I-III, respectively), we are able to reduce those uncertainties which are due to the inhomogeneity of the cluster parameters, and we can better estimate biases due to an incompleteness of the cluster sample. The COCD is originated from the All-Sky Compiled Catalogue of 2.5 million stars (ASCC-2.52; Kharchenko 2001) including absolute proper motions in the Hipparcos system, $B, V$ magnitudes in the Johnson photometric system, and supplemented with spectral types and radial velocities if available. The ASCC-2.5 is complete up to about $V=11.5 \mathrm{mag}$. We identified $520 \mathrm{of}$ about 1700 known clusters (Paper I) in the ASCC-2.5 and found 130 new open clusters (Paper III). Therefore, the completeness of the cluster sample is mainly defined by the limiting magnitude of the ASCC-2.5. For each cluster, membership was determined by use of spatial, kinematic, and photometric information (Paper I), and a homogeneous set of structural, kinematic and evolutionary parameters was obtained by applying a uniform technique (Papers II and III). The sample was used to study the population of open clusters in the local Galactic disk by jointly analysing the spatial and kinematic distributions of clusters (Piskunov et al. 2005, Paper IV).

In this paper we use the homogeneous data on structural parameters of open clusters from the COCD to study general correlations including cluster sizes as well as to analyse the spatial distribution of cluster members from the point of view of mass segregation. In Sect. 2 we briefly describe the data set and estimate the statistic properties of the cluster sample. The relations between cluster radius and its location in the Galaxy are discussed in Sect. 3. The correlations of cluster size with age is considered in Sect. 4. In Sect. 5 we examine the effect of mass segregation in open clusters. A summary is given in Sect. 6 .

\section{Data}

The present study is based on the Catalogue of Open Cluster Data (COCD) and its Extension 1 described in Papers II and III. The complete sample consists of 650 objects of which 641 are open clusters and 9 are compact associations. For each COCD object, the catalogue contains celestial position, distance, reddening, age, angular sizes (core and cluster radii), and kinematic data (proper motions and, if available, radial velocity). The cluster parameters were obtained from a uniform data set (the ASCC-2.5 catalogue) and by use of a uniform technique of membership and parameter determination (Papers I and III).

\subsection{The completeness of the sample}

In Paper IV we estimated the completeness limit of our cluster sample to be $0.85 \mathrm{kpc}$. This result is based on the analysis of the surface density of open clusters in the Galactic plane as a

\footnotetext{
1 ftp://cdsarc.u-strasbg.fr/pub/cats/J/A+A/438/1163, ftp: //cdsarc.u-strasbg.fr/pub/cats/J/A+A/440, 403

2 ftp://cdsarc.u-strasbg.fr/pub/cats/I/280A
} 


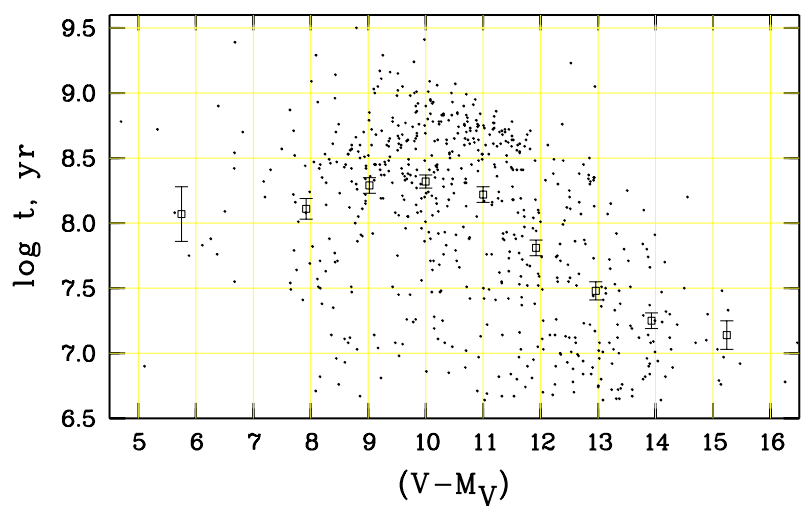

Fig. 1. Cluster age versus distance modulus. Dots are individual clusters. Their mean ages together with rms errors are shown as squares with bars.

function of their distance from the Sun. Nevertheless, for a statistical study of cluster sizes the distance modulus $\left(V-M_{V}\right)$ is, in many cases, a more suitable parameter than a distance itself: due to the expected effect of mass segregation, more massive (i.e. more luminous) cluster members are concentrated to the cluster centre, whereas fainter stars are located at the cluster borders. Therefore, the apparent size of a cluster depends on the brightness limit of the input catalogue, as well as on the distance and extinction for a given cluster (i.e. on $\left(V-M_{V}\right)$ ). Since reddening is known for each cluster of our sample, we compute a typical distance modulus $\left(V-M_{V}\right)=5 \log d-5+3.1 \times E(B-V)$ for clusters at $0.85 \mathrm{kpc}$ from the Sun to be $10.0-10.5 \mathrm{mag}$. Taking into account the completeness limit of the ASCC-2.5 at $V=11.5$, the corresponding limit in absolute magnitudes of cluster members is about 1.5...1.0 mag. This absolute magnitude is still sufficiently faint to observe MS stars in clusters younger than $1 \mathrm{Gyr}$, whereas older clusters can be identified from their red giants. At distances larger than the completeness limit we are steadily losing old clusters, and our sample should get "younger" on average. Indeed, the completeness limit at $\left(V-M_{V}\right) \approx 10.0-10.5$ can be clearly concluded from Fig. 1 where we show the distribution of cluster ages versus distance modulus.

\subsection{Linear sizes of open clusters}

For all clusters of our sample, we determined/redetermined the angular sizes by applying the same method (see Paper II for details). This approach is based on an iterative procedure which includes simultaneous determination of membership and parameters for a cluster. The selection of members takes into account photometric (location in the CMD) and astrometric (proper motions and positions) criteria, and the standard output parameters are the coordinates of the cluster centre, angular size, mean proper motion, distance, extinction, and age.

Based on stellar counts, we considered two empirical structural components for each cluster, the core and the corona (Paper II). The distribution of $1 \sigma$-members (i.e. stars with the membership probability $P \geq 61 \%$ ) was the most important factor for the determination of the cluster radius. The core radius was defined as the distance from the cluster centre where the decrease of stellar surface density gets flatter. The corona radius (or simply, cluster radius) corresponds to a distance where the surface density of stars becomes equal to the average density of the surrounding field.

The linear radii were computed from the individual distances and angular sizes of the clusters. General properties of their
Table 1. Radii (in pc) of clusters $\left(R_{\mathrm{cl}}\right)$ and associations $\left(R_{\mathrm{ass}}\right)$. Standard deviations are given in brackets.

\begin{tabular}{lccc}
\hline \hline & \multicolumn{2}{c}{ New } & Lyngå \\
\cline { 2 - 3 } & Core & Corona & $(1987)$ \\
\hline$\overline{R_{\mathrm{cl}}}(10$ smallest $)$ & $0.5(0.1)$ & $0.9(0.2)$ & $0.5(0.3)$ \\
$\overline{R_{\mathrm{cl}}}(10$ largest $)$ & $5.8(2.4)$ & $16.8(2.4)$ & $6.1(4.9)$ \\
$\overline{R_{\mathrm{cl}}}(d<450 \mathrm{pc}, N=67)$ & $1.6(1.3)$ & $5.1(4.3)$ & $3.6(3.8)$ \\
$\overline{R_{\mathrm{cl}}}($ all,$N=510)$ & $2.0(1.3)$ & $5.0(3.2)$ & $2.7(2.7)$ \\
$\overline{R_{\mathrm{ass}}}($ all,$N=9)$ & $6.9(2.3)$ & $33.2(21.7)$ & - \\
\hline
\end{tabular}

distribution are given in Table 1. For about 500 open clusters of our sample, the sizes are also given in Lyngå (1987). As expected (see also Paper II), the cluster radii from Lyngå (1987) are in average lower by a factor of 2 , and they fit rather the core than the corona. Here we would like to stress the main advantage of our approach with respect to other methods: our estimations of cluster sizes are based on complete information on cluster membership which includes the photometric as well as astrometric criteria. In order to illustrate, as an example, the importance of reliable membership data for a cluster parameter determination, we refer the reader to the cluster Ruprecht 147 (cf. COCD, Atlas, p. 460) which is included in the catalogue of Lyngå (1987). Neither distance nor age was known for this cluster, and a diameter of 47 arcmin was estimated. Applying our procedure of membership and parameter determination, we found that the cluster has a proper motion $\left(\mu_{\delta}=27.7\right.$ mas $\left./ y r\right)$ which differs very strongly from the field. It turns out that Ruprecht 147 is an old cluster $(\log t=9.39)$, at $175 \mathrm{pc}$ from the Sun with an angular diameter of about 2.5 degrees i.e., 3 times larger than in Lyngå (1987).

\subsection{Statistic properties of the cluster sample}

In order to find statistical relations involving sizes of open clusters, we excluded 9 objects from our original sample since they are generally accepted to be associations. Further, the two closest clusters, the Hyades and Collinder 285 (the UMa cluster), are missing in our list. Since they occupy large areas on the sky, a specific technique of membership determination is required for them. Although the parameters needed can be obtained from published data, we prefer, from the point of view of data homogeneity, not to include these clusters in the sample. So, the total cluster sample contains 641 open clusters.

Compared to previous work, this is the largest sample of galactic open clusters ever used to find out statistical correlations including cluster sizes. Studying the properties of open clusters, Lyngå (1982) and Janes et al. (1988) were limited to samples of about 400 objects, mainly due to a lack of ages and/or distances for clusters. The sample of Tadross et al. (2002) includes 160 clusters for which $U B V$ CCD observations are available. Although some of the cluster parameters were redetermined by the authors, the samples are compilations of published data and therefore, they are neither complete nor homogeneous. From this point of view, a reliable statistical proof of apparent correlations is rather difficult.

It is obvious, that the quantitative expressions derived below in Sects. 3 and 4 depend strongly on the conventions used for the definition of cluster sizes. Thanks to the homogeneity of the data, it is expected that significant correlations, derived with these data, indicate real trends. Nevertheless, studying the distribution of cluster sizes, we must take into account biases due 


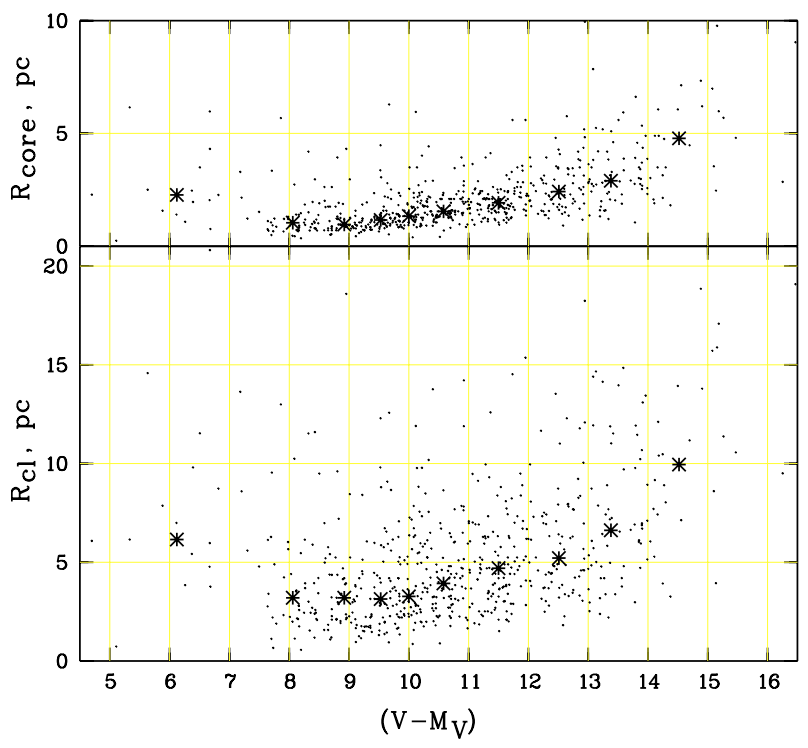

Fig. 2. Linear radius of open clusters (bottom) and of their cores (top) versus distance modulus. Dots are individual clusters, asterisks mark the corresponding medians in bins of distance moduli.

to the relatively bright completeness limit of the ASCC- 2.5 at about $V=11.5$. These biases occur in any kind of magnitudelimited surveys, but in our case they become significant at relatively small distances. The first one, an apparent "rejuvenation" of the cluster sample with increasing distance from the Sun, is illustrated in Fig. 1 and discussed in Sect. 2.1.

Second, due to the low density of bright stars (about 150 stars/sq.deg in the Galactic plane, Høg et al. 2000), we could not resolve open clusters with an angular radius smaller than 0.08 degrees: one of our criteria for the detection of a cluster was the presence of at least three $1 \sigma$-members in already known clusters and at least 8 members in newly detected clusters. This is the reason for the absence of small clusters at large distances in Fig. 2. Provided that the frequency of small clusters at large distances is comparable to what we observe within $400 \mathrm{pc}$ from the Sun, there are about 10-15 clusters (i.e., 4-6\%) still to be discovered within $850 \mathrm{pc}$. Of course, the number of missing clusters grows rapidly with distance, and our sample becomes more and more biased towards large young clusters (cf. Fig. 2).

The other bias in the determination of sizes arises due to expected effect of mass segregation in open clusters. This means that at larger distances we do not see faint members located in outer regions of a cluster and thus we will systematically underestimate its size. This bias is also a function of the limiting magnitude of the input catalogue as well as of distance (or, more precisely, of the distance and interstellar extinction, i.e. of distance modulus) of a cluster, and its influence is difficult to estimate quantitatively.

Finally, since linear sizes of clusters are derived from angular sizes and distances, their accuracy decreases with increasing distance. If not taken into account, this obvious fact may lead to a misinterpretation of the apparent distribution of linear sizes.

Assuming that these biases affect the size determination of all clusters in a similar way, we can, however, study the distribution of linear radii of different cluster groups provided that they have a comparable distribution with respect to their distance moduli. In this case the impact of biases onto the solution can be better taken into account.

\section{Relations between cluster size and the location in the Galaxy}

Throughout the paper we use the rectangular coordinate system $X, Y, Z$ with origin in the barycentre of the Solar system, and axes pointing to the Galactic centre $(X)$, to the direction of Galactic rotation $(Y)$, and to the North Galactic pole $(Z)$. Galactocentric distance $\left(R_{\mathrm{G}}\right)$ and distance from the symmetry plane $\left(\left|Z^{\prime}\right|\right)$ are computed for each open cluster under the assumption that the Sun is located $8.5 \mathrm{kpc}$ from the Galactic centre and $22 \mathrm{pc}$ above the symmetry plane of the cluster system (Paper IV).

\subsection{Cluster radius versus Galactocentric distance}

The discussion on a possible dependence of the linear sizes of open clusters from the Galactocentric distance has a long and controversial history. Considering the sample of 150 open clusters from the Becker \& Fenkart (1971) catalogue, Burki \& Maeder (1976) concluded that the size of the youngest clusters $(\log t<7.20)$ increases with distance from the Galactic centre. Based on a sample of about 400 clusters, Lyngå (1982) found that large clusters $\left(R_{\mathrm{cl}}>5 \mathrm{pc}\right)$ are mainly located outside the Solar orbit. Using the same sample but with redetermined sizes and distances for clusters, Janes et al. (1988) did not reveal any significant relation between cluster size and Galactocentric distance. With a sample of 160 open clusters, Tadross et al. (2002) found a correlation between cluster size and Galactocentric distance for clusters over the whole range of ages, whereas Nilakshi et al. (2002) could confirm an increase of cluster sizes at $R_{\mathrm{G}}>$ $9.5 \mathrm{kpc}$.

Since we are interested to find a possible gradient of linear cluster sizes as a function of the Galactocentric distance, we exclude clusters with $|Y|>2 \mathrm{kpc}$. Although at large distances from the Sun, those clusters may have a Galactocentric distance comparable to that of the Sun. In this case they do not contribute effectively to the analysis but introduce an additional noise due to uncertain parameters. Moreover, considering a stripe along the Galactic radius, we are safer to assume that the biases described in Sect. 2.3 are symmetrical to the Sun's location, and possible differences in the sizes of clusters for the inner $\left(R_{\mathrm{G}}<8.5 \mathrm{kpc}\right)$ and outer $\left(R_{\mathrm{G}}>8.5 \mathrm{kpc}\right)$ disk should be real, if found in the first place.

In order to compare the distributions of linear sizes of clusters in the inner $(N=310$ clusters $)$ and outer $(N=270$ clusters) disk, a Kolmogorov-Smirnov $(K-S)$ test was applied. We determined a probability $p=0.002$ for the null hypothesis that the linear radii of "inner" and "outer" subsamples are drawn from the same distribution. Further $K-S$ tests showed that the differences in the distributions were caused by clusters with age $\log t>8.35$. The relation between cluster radius and Galactocentric distance is shown in Fig. 3 for four different age groups of clusters. The corresponding least-square regression lines were calculated for clusters with $7<\left(V-M_{V}\right)<12$. This region was chosen as a compromise: on one hand, an $R_{\mathrm{G}}$ spread had to be kept as large as possible since we looked for a large scale effect; on the other hand, an impact of the biases (see Fig. 2) must be minimised. Provided that the biases have a similar influence on "inner" and "outer" subsamples, the compromise is acceptable.

The age limits were not chosen arbitrarily. Analysing the kinematics of open clusters in Paper IV, we derived a rotation velocity of the cluster system of $234 \mathrm{~km} \mathrm{~s}^{-1}$ at the Galactocentric distance of the Sun which corresponds to a rotation period $P_{\mathrm{GR} 0}$ 


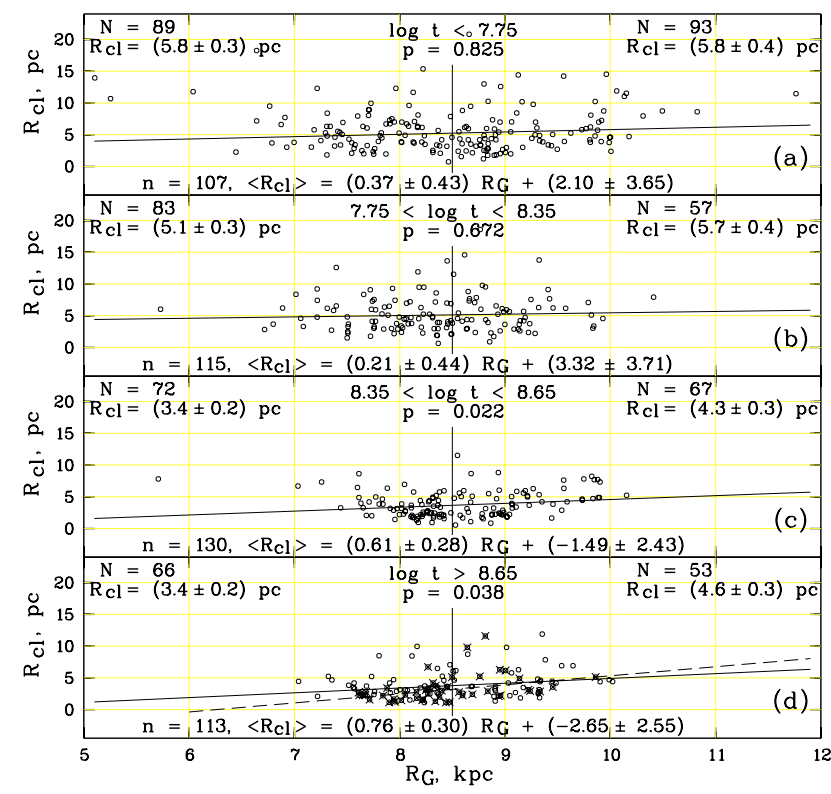

Fig. 3. Linear radius of clusters versus Galactocentric radius $\left(R_{\mathrm{G}}\right)$ for four age groups as indicated at the top of each panel. Circles mark all clusters with $|Y|<2 \mathrm{kpc}$. The line at $R_{\mathrm{G}}=8.5 \mathrm{kpc}$ divides clusters into "inner" and "outer" subsamples. For each subsample, $N$ gives the number of clusters, $R_{\mathrm{cl}}$ is the mean cluster radius. $p$ is the probability that "inner" and "outer" clusters stem from the same statistical sample. The solid lines are regression lines fitting the observed distributions of the linear radii for clusters within $7<\left(V-M_{V}\right)<12$. The parameters of the regression lines and the number of clusters included in the solution are shown at the bottom of the corresponding panel. For panel d) crosses mark clusters older than $\log t=8.85$, and the broken line is the corresponding regression line.

of about 225 Myr around the Galactic centre. The youngest group $(\log t \leq 7.75)$ includes clusters younger than $0.25 P_{\mathrm{GR} 0}$. The clusters of the groups (b) and (c) have ages from $0.25 P_{\mathrm{GR} 0}$ to $1 P_{\mathrm{GR} 0}$ and from $1 P_{\mathrm{GR} 0}$ to $2 P_{\mathrm{GR} 0}$, respectively; whereas the cluster in group (d) are older than $2 P_{\mathrm{GR} 0}$. Although the selection effects and biases are clearly seen (no small clusters at larger distances from the Sun, no old clusters at distances larger than $1.5 \mathrm{kpc}$ from the Sun), they affect the "inner" and "outer" cluster subsamples in a similar way. Whereas the younger cluster groups do not show any significant correlation between their size and Galactocentric distance, a probable dependence appears after the first revolution around the Galactic centre and becomes significant (at 2.5 $\sigma$-level) when clusters passed (survived) two revolutions. The old clusters in the inner disk are on average smaller $\left(\overline{R_{\mathrm{cl}}}=3.8 \pm 0.2 \mathrm{pc}\right)$ than in the outer disk $\left(\overline{R_{\mathrm{cl}}}=4.6 \pm 0.3 \mathrm{pc}\right)$, and the probability that both cluster groups stem from the same statistical sample is less than $4 \%$.

According to Friel (1995) no clusters older than $\log t=$ 8.9 Myr (about the age of the Hyades) have been found within $7.5 \mathrm{kpc}$ from the Galactic centre. Our data support this result, though we cannot exclude the possibility that some small old clusters could be located at smaller $R_{\mathrm{G}}$ and will be found with deeper surveys in the future. They may not have been discovered yet, due to their small size and low contrast to the field, or due to clouds in the line of sight. Extrapolating the relation between cluster size and galactocentric radius derived for the old clusters (Fig. 3, panel (d)), we conclude that for old clusters $(\log t>8.65, \overline{\log t}=8.86)$, the limiting distance from the Galactic centre should be about $3.5 \mathrm{kpc}$. By continously excluding younger clusters from the subsample (d), we obtained steeper and steeper slopes of the relation and larger limiting radii $R_{\mathrm{G}}$. For $\log t>8.85(\overline{\log t}=9.02)$, the limiting Galactocentric radius is about $6.3 \mathrm{kpc}$. Although the number of clusters included in the latter case is relatively small $(N=45)$, the slope is significant $\left(\overline{R_{\mathrm{cl}}}=(1.42 \pm 0.49) R_{\mathrm{G}}-(8.90 \pm 4.16)\right)$. In other words, no clusters older than $1 \mathrm{Gyr}$ should exist at galactocentric radius less than $6 \mathrm{kpc}$.

\subsection{Cluster radius versus distance from the symmetry plane of the cluster system}

Considering the sizes of clusters as a function of the distance from the Galactic plane, Janes et al. (1988) found that small clusters show a stronger concentration to the Galactic plane, independent of age. A few large clusters are either old clusters at larger distances from the Galactic plane or young clusters located close to the Galactic plane. Tadross et al. (2002) came to similar conclusions, whereas no correlation between cluster sizes and $Z$ coordinates was found by Nilakshi et al. (2002). Lyngå (1982) presented a figure showing a distribution of cluster ages versus distance from the Galactic plane, and he also distinguished between different cluster sizes. No dependence between sizes and distances from the Galactic plane is visible in this figure, and Lyngå (1982) did not comment this issue. We should note that large old clusters from the samples of Janes et al. (1988) and Tadross et al. (2002) are located at such distances from the Sun (mainly from $1 \mathrm{kpc}$ to $4 \mathrm{kpc}$ ) where the cluster samples are highly biased by incompleteness. Hence, an absence of small clusters at large $Z$ distances could be real or simply an apparent trend due to selection effects and/or biases.

In order to minimise correlations due to different $R_{\mathrm{G}}$ and the biases described above, we consider clusters with $7.0<$ $\left(V-M_{V}\right)<10.5$, i.e. in a $\left(V-M_{V}\right)$ range where our sample is practically complete. Although the mean cluster size $R_{\mathrm{cl}}$ is computed as $4.1 \pm 0.2 \mathrm{pc}$, the clusters at $\left|Z^{\prime}\right|<50 \mathrm{pc}$ are, on average, smaller $\left(R_{\mathrm{cl}}=3.6 \pm 0.2 \mathrm{pc}\right)$ than those at $\left|Z^{\prime}\right|>100 \mathrm{pc}$ $\left(R_{\mathrm{cl}}=5.2 \pm 0.4 \mathrm{pc}\right)$. The distribution of cluster sizes versus distance from the symmetry plane (where $Z^{\prime}=0$ ) is shown in Fig. 4 for different ages. According to Fig. 4, a systematic trend of cluster sizes with increasing $\left|Z^{\prime}\right|$ can be observed for all clusters in the Solar vicinity, and this correlation becomes highly significant for clusters older than $\log t>8.35$, which already survived at least one rotation around the Galactic centre.

\subsection{Cluster radius versus Galactocentric radius and distance from the symmetry plane of the cluster system}

In order to check whether a multi-parameter correlation can be observed for cluster sizes in our data set, we considered the same subsamples as in Sect. 3.1. The distribution of $\left|Z^{\prime}\right|$ distances versus Galactocentric radius $R_{\mathrm{G}}$ is shown in Fig. 5. For each age group we computed the mean cluster radius and the corresponding standard deviation. We call the clusters "large" or "small" if their linear radii differ from the corresponding mean by at least one standard deviation. These clusters are indicated in Fig. 5 by different symbols. As expected, the youngest clusters (a) are more strongly concentrated to the plane of the symmetry than the oldest clusters (d). Small and large clusters of the youngest group show a similar distribution with $R_{\mathrm{G}}$ as well as with $\left|Z^{\prime}\right|$. The location of young clusters is not uniform in the $R_{\mathrm{G}}-\left|Z^{\prime}\right|$ space but shows links of the clusters to their formation places rather than any systematics. In contrast, the spatial distributions 


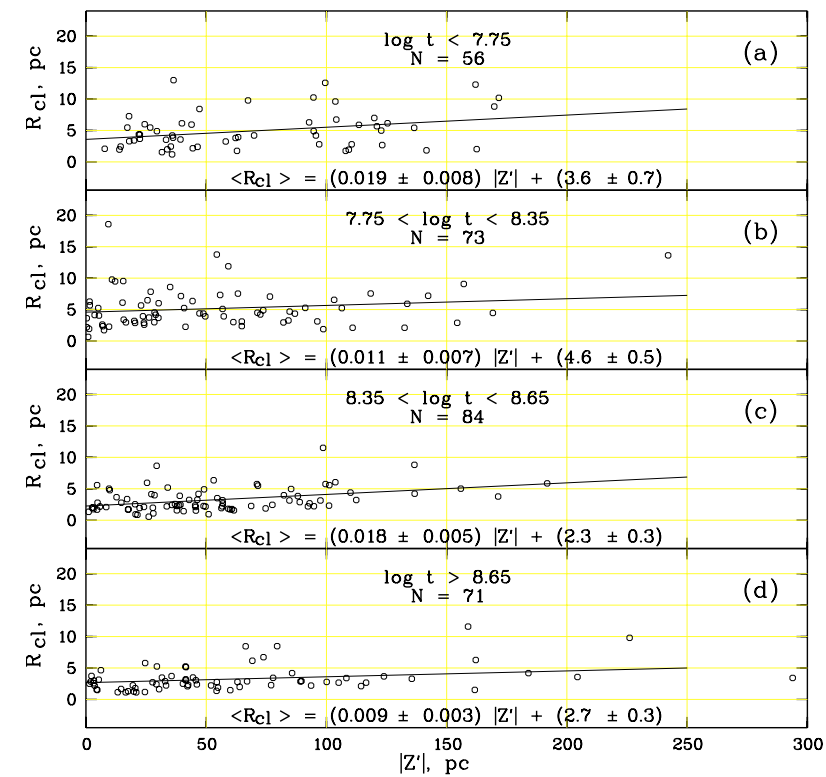

Fig. 4. Linear radius of clusters versus distance from the symmetry plane of the cluster system for four age groups as indicated at the top of each panel. Circles mark all clusters with $7.0<\left(V-M_{V}\right)<10.5$. $N$ gives the number of clusters in each subsample, and the solid line is the corresponding regression line fitting the observed distributions. The parameters of the regression lines are given at the bottom of each panel. Note on panel d) the cluster NGC 2682 with $\left|Z^{\prime}\right|=502 \mathrm{pc}$ is not shown though it was included into the regression calculation.

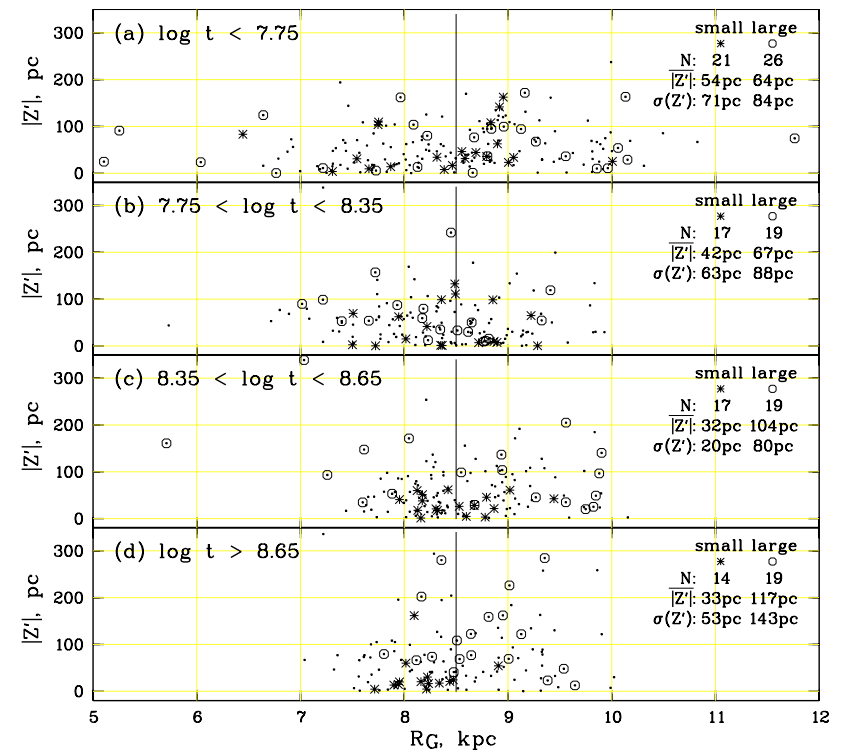

Fig. 5. Distance of open clusters from the symmetry plane versus Galactocentric radius for four age groups as indicated at the top of each panel. Dots mark all clusters with $|Y|<2 \mathrm{kpc}$. Circles are "large" clusters, asterisks mean "small" clusters. For the definition of "large" and "small" see text. In each panel, $N$ is the number of "small" or "large" clusters, $\overline{\left|Z^{\prime}\right|}$ is the mean distance from the symmetry plane, and $\sigma\left(Z^{\prime}\right)$ is the dispersion of $Z^{\prime}$. The line at $R_{\mathrm{G}}=8.5 \mathrm{kpc}$ divides clusters into "inner" and "outer" subsamples.

of small and large clusters differ in the oldest cluster group. The small old clusters are more concentrated to the symmetry plane, whereas large old clusters are found at large distances from this plane, though the spread of $Z^{\prime}$ coordinates is large, too. Except in one case, all large old clusters are at $R_{\mathrm{G}}>8 \mathrm{kpc}$. Such a

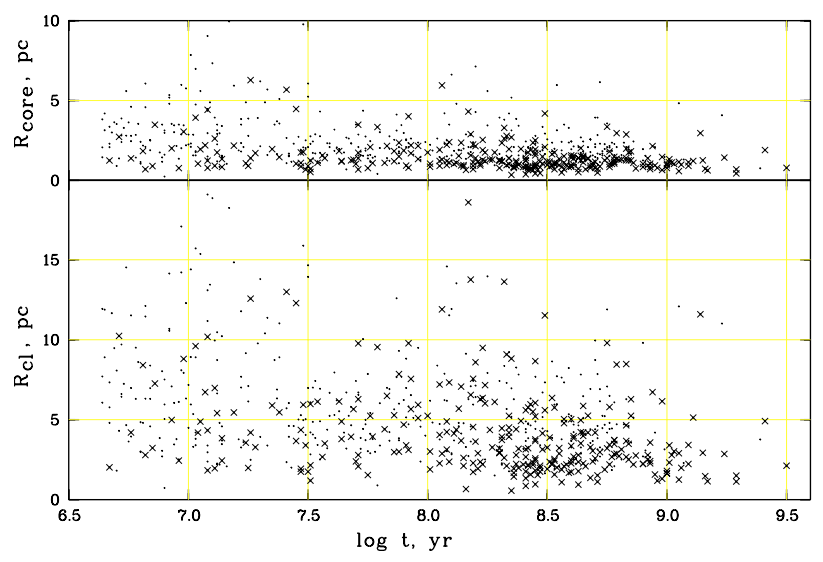

Fig. 6. Linear radius of open clusters (bottom) and of their cores (top) versus cluster ages. Crosses mark clusters with $7.0 \leq\left(V-M_{V}\right) \leq 10.5$.

distribution would appear if the interstellar extinction were much stronger at $7.5<R_{\mathrm{G}}<8.0$ than at $9.0<R_{\mathrm{G}}<9.5$. In this case, one would expect to see this effect for other age groups, also. Since we do not observe a similar evidence for younger clusters, we conclude that the absence of large old clusters at smaller $R_{\mathrm{G}}$ indicate a real trend.

Summarising the findings of this section, we propose the following scenario for cluster evolution within the Solar neighbourhood. Clusters are formed within a thin disk inside as well as outside the Solar orbit. Their initial sizes do not show significant correlations with the $R_{\mathrm{G}^{-}}$and $\left|Z^{\prime}\right|$ - coordinates. After one revolution around the Galactic centre, the distribution of cluster sizes becomes more systematic with respect to the cluster location: small clusters are more probably found at low $Z^{\prime}$ coordinates, whereas large clusters are generally located at larger $Z^{\prime}$ and/or at larger $R_{\mathrm{G}}$. Some of the large young clusters probably dissolve during the first revolution around the Galactic centre. However, they have a higher chance to survive encounters with molecular clouds and the impact of Galactic tidal forces and reach higher ages if their orbits are outside the Solar orbit and are inclined to the Galactic plane (cf. Fig. 5). The concentration of small clusters with $R_{\mathrm{G}}<8.5 \mathrm{kpc}$ to the symmetry plane supports indirectly the conclusions drawn from the simulations by Spitzer \& Chevalier (1973) that compact clusters survive against external shocks for a longer time.

\section{Relations between cluster size and age}

Neither Lyngå (1982), Janes et al. (1988), Tadross et al. (2002) nor Nilakshi et al. (2002) found a correlation between cluster sizes and ages. This is not very surprising since, as discussed in the previous section, cluster sizes seem to show a multiparametric dependence. The separation of different effects is rather difficult, especially, if one cannot rely on a complete and unbiased sample as well as on the homogeneity of cluster parameters. Nevertheless, the relations between the linear radii and the location of open clusters in the Galaxy indicate a correlation with cluster age. This gives us a strong hint to look more carefully whether any direct dependence of cluster sizes on age can be found in our data. Since the age for each open cluster of our sample was determined by the same method and linear sizes $R_{\mathrm{cl}}$, $R_{\text {core }}$ were derived via of the same conventions, the data provide the best preconditions for checking whether real trends in structural parameters exist, depending on ages. 


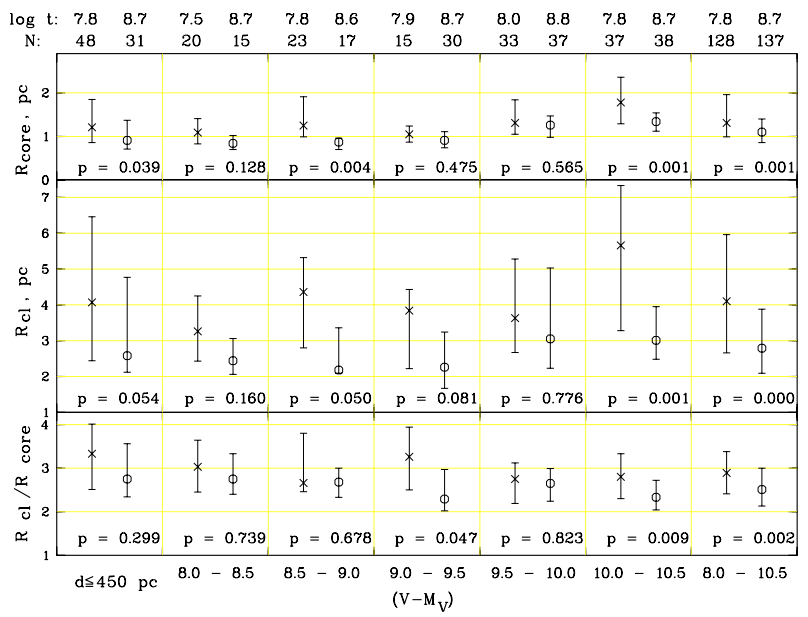

Fig. 7. Structural parameters of open clusters for two age groups: a young group with $\log t \leq 8.4$, and an old group with $\log t>8.4$. The upper panel is for cluster core radii $R_{\text {core }}$, the central - for linear radii of clusters $R_{\mathrm{cl}}$, and the lower - for ratios $R_{\mathrm{cl}} / R_{\text {core }}$. Crosses and circles show the median for young and old groups, respectively, and bars are $Q_{1}, Q_{3}$ quartiles computed for each age group and for different $\left(V-M_{V}\right)$ bins (as indicated at the bottom). $\log t$ and $N$ at the top give the average age and the number of clusters in the corresponding subsample, and $p$ is the probability that the parameters of young and old groups at a given $\left(V-M_{V}\right)$ are drawn from the same distribution.

The distribution of clusters as a function of age is shown in Fig. 6. At the first glance, a decrease of cluster sizes and their scattering with age may be suggested, though, the majority of large-radii clusters are distant young ones. In order to minimise possible biases described above, we selected clusters within 5 small bins of distance moduli $\Delta\left(V-M_{V}\right)=0.5$ and considered the linear radii of clusters in two subgroups, younger and older than $250 \mathrm{Myr}(\log t=8.4)$. This age limit was chosen arbitrarily, only to provide more or less comparable numbers of clusters in young and old groups. Additionally, we considered all clusters within the range $\left(V-M_{V}\right)=8 \ldots 10.5$ as well as the nearby clusters with distances up to $450 \mathrm{pc}$ from the Sun. Since the cluster radii are not distributed normally but show rather skewed distributions with long tails towards large radii, the mean values of the subsamples are affected by extreme values of "outliers". The impact can be essential since the number of clusters in each subsample is relatively small. Therefore, we considered the median, the first $Q_{1}$ and third $Q_{3}$ quartiles of each data set. The results are given in Fig. 7. Applying the $K-S$ test, we also computed the probabilities $p$ for the null hypothesis that the linear radii of young and old groups at a given $\left(V-M_{V}\right)$ are drawn from the same distribution.

Although the cluster sizes $R_{\mathrm{cl}}$ of two age groups do not always differ significantly (i.e., $p>0.05$ ), the general tendency remains remarkably constant: the younger groups have in average larger sizes by a factor of $\approx 1.6(1.2 \ldots 2.0)$, and they show a larger spread of sizes. This is also valid for the cluster cores, though, the effect is smaller. The ratios $R_{\mathrm{cl}} / R_{\text {core }}$ are more affected by poor statistics, though they also indicate a similar tendency: their medians range within $2.7 \ldots 3.3$ for the younger groups, and within $2.3 \ldots 2.7$ for the older clusters. We conclude that the results seem to indicate trends in structural parameters depending on cluster ages.

In order to obtain a quantitative expression for the dependence of cluster sizes on the age and to check its significance, the clusters were divided into 5 age groups with $\log t$ (i.e., $\leq 7.50$, $7.50 \ldots 8.00,8.00 \ldots 8.45,8.45 \ldots 8.80,>8.80)$. Again, these ranges

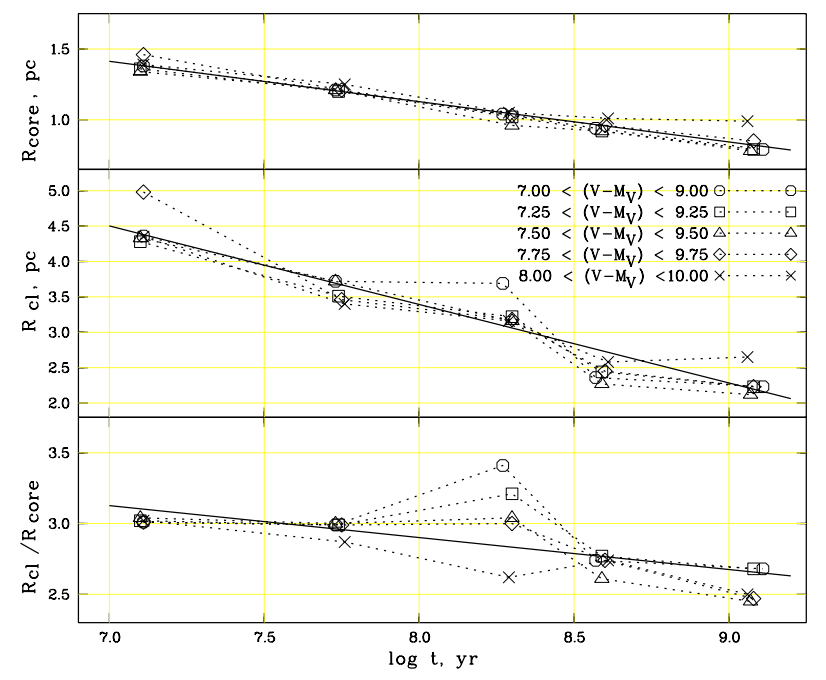

Fig. 8. Structural parameters of open clusters versus age. The upper panel is for linear radii of cluster cores $R_{\text {core }}$, the central - for linear radii of clusters $R_{\mathrm{cl}}$, and the lower - for the ratios $R_{\mathrm{cl}} / R_{\text {core }}$. Thin dashed lines connect the medians determined for different age groups within one $\left(V-M_{V}\right)$-bin. The solid lines are the regression lines fitting the observed distributions of the medians.

were chosen arbitrarily, as a compromise between the number of age groups and numbers of clusters in each age group. Further, we selected clusters with $\left(V-M_{V}\right)$ from 7 mag to 10 mag binned in 5 overlapping groups (see Fig. 8). In each age $/\left(V-M_{V}\right)_{i}$ subsample, the median is determined for $R_{\text {core }}, R_{\mathrm{cl}}$, and $R_{\mathrm{cl}} / R_{\text {core }}$. The corresponding $5 \times 5$ realisations of the medians are shown in Fig. 8. The resulting relations between structural parameters of open clusters and their ages $\log t$ can be approximated by the following equations:

$$
\begin{aligned}
R_{\mathrm{cl}} & =(-1.11 \pm 0.08) \log t+(12.26 \pm 0.67) \\
R_{\text {core }} & =(-0.28 \pm 0.02) \log t+(3.40 \pm 0.12) \\
R_{\text {cl }} / R_{\text {core }} & =(-0.23 \pm 0.06) \log t+(4.70 \pm 0.44)
\end{aligned}
$$

where $R$ is measured in parsec. For comparison, we recomputed the dependences by including all 209 clusters within $\left(V-M_{V}\right)$ from 7 mag to 10 mag:

$$
\begin{aligned}
R_{\mathrm{cl}} & =(-1.26 \pm 0.30) \log t+(14.35 \pm 2.49) \\
R_{\text {core }} & =(-0.39 \pm 0.09) \log t+(4.54 \pm 0.78) \\
R_{\mathrm{cl}} / R_{\text {core }} & =(-0.26 \pm 0.10) \log t+(5.09 \pm 0.84)
\end{aligned}
$$

Due to "outliers" (i.e., the clusters with large $R_{\mathrm{cl}}$ and $R_{\text {core }}$ ), the regression lines are somewhat shifted but the differences are still within the error range. Although all terms in Eqs. (1), (2) are highly significant, the derived relations are, of course, not universal. Based on other definitions of $R_{\mathrm{cl}}$ and $R_{\text {core }}$ or on a survey with different completing magnitude, the relations may change. One may expect that the impact would be stronger on the "zeropoint"-term than on the slopes, describing the correlation of radii with age which are, indeed, the more important and interesting parameters. According to Eqs. (1), (2), we conclude that, on average, the apparent linear sizes of clusters and their cores are decreasing with time and that the process is going faster for the cluster sizes themselves than for their cores. The question is whether the averaged size of open clusters really become smaller with age, or if this is an apparent trend due to e.g. mass segregation effects acting differently in old and young clusters, or perhaps there is a mixture of both effects. 


\section{Mass segregation in open clusters}

In order to quantify the effect of mass segregation in open clusters, different approaches are usually applied. The majority of methods is based on the comparison of the integrated profiles of the surface density for stars with different mass, and on an analysis of the differences in their concentration to the cluster centre (e.g., Mathieu 1984; Sagar et al. 1988; Raboud \& Mermilliod 1998a,b; Hillenbrand \& Hartmann 1998; Bonatto \& Bica 2005). Another approach considers luminosity and/or mass functions and compares their slopes for cluster stars located in the central and outer areas of clusters (Fischer et al. 1998; de Grijs et al. 2002a,b,c). This method is indirect and rather difficult to apply, since a survey of identical completeness is required for the central and outer regions. Usually, this requirement is hardly achievable due to crowding effects in the cluster center. Some authors (Sagar et al. 1988; Hillenbrand \& Hartmann 1998; Slesnick et al. 2002) consider radial trends in the average stellar mass, though this method needs not only a complete but also a deep survey due to the weak significance of the effect.

Due to the relatively bright limiting magnitude of the ASCC-2.5, and therefore, the relatively low average number of the most probable members we cannot apply the methods described above to the majority of clusters of our sample. Therefore, we need to introduce another parameter which takes into account properties of our data. Then, having the uniform data set of linear sizes and the information on spatial distribution of the most probable members in each cluster, we can analyse the effects of mass segregation and study general trends in the mass distribution of members in open clusters of different ages.

For an easier interpretation of the results on mass segregation, we computed masses of cluster members within a range of absolute magnitudes which is typical for our sample. We used the Padova grid of overshooting isochrones (Girardi et al. 2002) with input parameters $m=0.15 \ldots 66 m_{\odot}, Z=0.019, Y=0.273$. As we consider open clusters in a relatively small range of distances from the Sun, a possible impact of metallicity variation on mass determination can be ignored. In order to avoid the strong uncertainty due to models for red giants, we did not include members to the right of the TAMS. In Fig. 9a we show a combined CMD of the most probable members of open clusters with $\left(V-M_{V}\right)<10.5$ and a number of isochrones covering the complete range of cluster ages in our sample. Figure $9 b$ gives the relation between absolute magnitude and stellar mass for cluster members located to the left of the TAMS in Fig. 9a. Consequently, the main sequence (MS) members of the clusters of our sample cover ranges $M_{V} \approx-6 \ldots+6 \mathrm{mag}$ and $\log m \approx 1.5 \ldots-0.1$, where $m$ is in units of solar masses.

Now we come back to the question at the end of the previous section. Considering only clusters within the completeness area, let us compare the correlations of sizes of the youngest and oldest clusters with their distance from the Sun. If one assumes a higher concentration of relatively massive stars (observable in the ASCC-2.5 up to large distances) to the cluster centre and a widely spread distribution of fainter stars (missing in the ASCC-2.5 at large distances), the linear sizes of open clusters should decrease with increasing $\left(V-M_{V}\right)$. If the sizes of the oldest clusters would decrease faster than the sizes of the youngest group, it would hint to a stronger mass segregation in older clusters. The distribution of cluster sizes is shown in Fig. 10 for young $(\log t<7.9)$ and old $(\log t>8.6)$ clusters with distance moduli $\left(V-M_{V}\right)<10.5$. Unfortunately, due to the relatively low spatial density of clusters, we are, in practice, limited to a $\left(V-M_{V}\right)$ range between 7.5 and 10.5 mag which, taking into

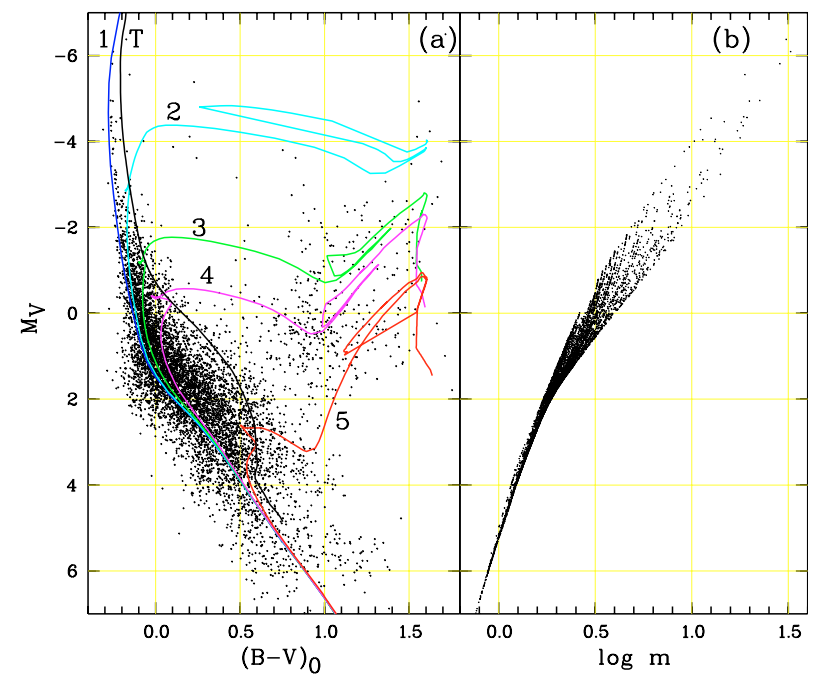

Fig. 9. Absolute magnitude $\left(M_{V}\right)$ and mass $(\log m)$ of the most probable members of clusters with $\left(V-M_{V}\right)<10.5$. Panel a) CMD. Curves 1, 2, 3,4 , and 5 show the Padova isochrones for $\log t=6.65,7.75,8.35,8.65$, and 9.50, respectively; the TAMS is marked by T. Panel b) Relation between absolute magnitude and $\log m$ of the most probable MS members. $m$ is in units of solar masses.

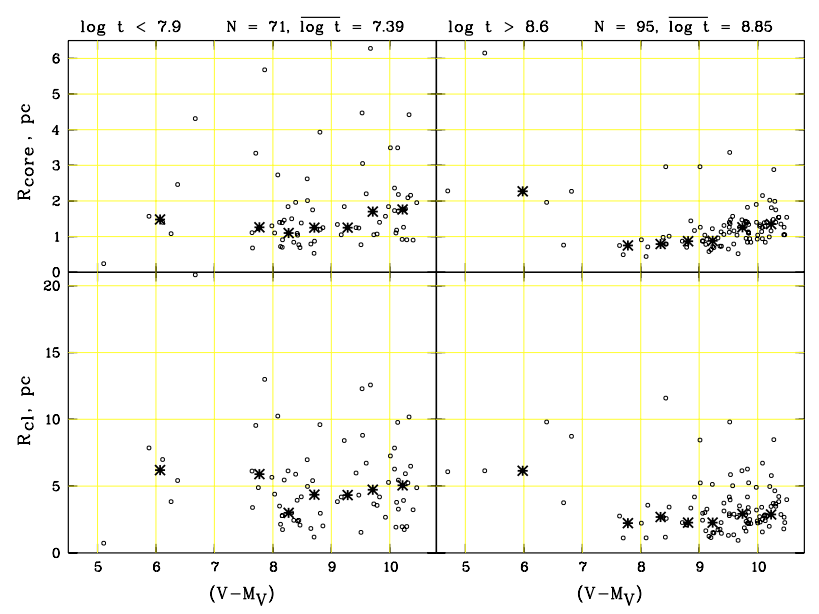

Fig. 10. Linear radii of clusters (bottom panels) and cores (upper panels) versus distance moduli for two different age groups as indicated at the top. Circles are individual clusters, asterisks show the corresponding medians of cluster and core radii. $N$ and $\overline{\log t}$ are the numbers of clusters in each group and their mean age.

account the large scattering of cluster sizes, is rather small to get a clear quantitative proof. Nevertheless, qualitative conclusions seem possible.

With the given completeness limit of the ASCC-2.5 at about $11.5 \mathrm{mag}$, the faintest stars, which we observe in a cluster at $\left(V-M_{V}\right)=6$, are about $M_{V}=5.5$ corresponding to $m \approx 0.9 m_{\odot}$. On the other hand, at $\left(V-M_{V}\right)=10.5$ the observed sizes of clusters are defined by stars brighter than $M_{V}=1$ with masses $m>2.5 m_{\odot}$. According to the median of radii, a few nearby clusters $\left(\overline{V-M_{V}} \approx 6\right)$ in Fig. 10 (bottom panels) have, on average, comparable sizes independent of their age. This can occur if stars with masses $m \approx 0.9 m_{\odot}$ are observed at distances from the cluster centre which are similar for relatively young $(\overline{\mathrm{age}} \approx 30 \mathrm{Myr})$ and old $(\overline{\operatorname{age}} \approx 800 \mathrm{Myr})$ clusters. The situation changes if 


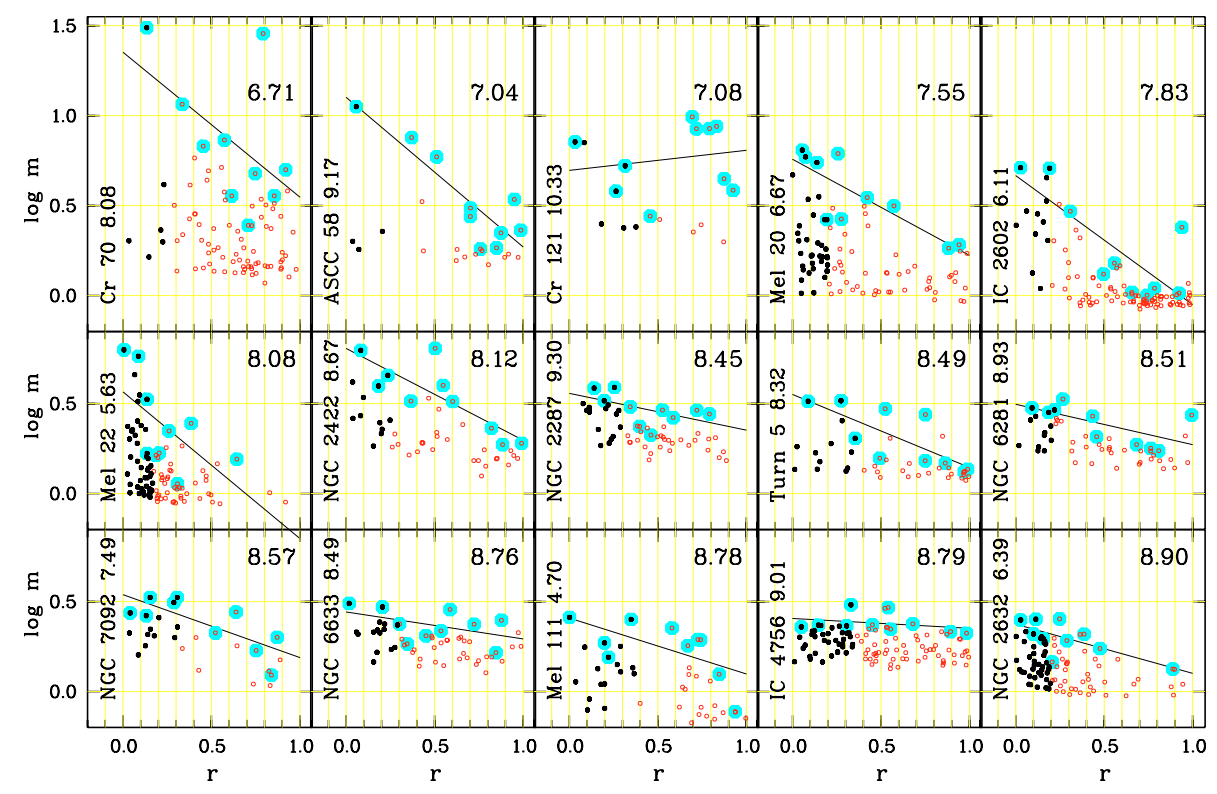

Fig. 11. The mass of the most probable members versus radial distance from the cluster centre (in units of $R_{\mathrm{cl}}$ ) for selected clusters of different ages. In each panel, the cluster name and distance modulus are shown on the left, whereas the cluster age is given on top. Black dots indicate cluster members projected onto the core area, (red) open circles mark members in the coronal area. Grey (cyan) circles show members included in the calculation of the corresponding regression shown as the straight line (see text for further explanation). Clusters are sorted with increasing age.

we consider stars of larger masses which are at the magnitude limit of the ASCC-2.5 at distance moduli between 8 and 10.5. In older clusters, the stars with masses between $1.3 m_{\odot}\left(M_{V}=3.5\right.$, $\left.\left(V-M_{V}\right)=8\right)$ and $2.6 m_{\odot}\left(M_{V}=1,\left(V-M_{V}\right)=10.5\right)$ are concentrated to the cluster center much stronger (by a factor of two) than stars of $0.9 m_{\odot}$ at $\left(V-M_{V}\right)=6$. On the other hand, this effect is less significant in young clusters: the cluster sizes defined by stars with masses $1.3 \ldots 2.6 m_{\odot}$ are only slightly smaller than the sizes defined by stars with masses $0.9 m_{\odot}$. On average, young clusters are 1.6 times larger than old clusters if we consider members with masses $1.3 \ldots 2.6 m_{\odot}$ at $\left(V-M_{V}\right)=8 \ldots 10.5$. Although the scattering of radii is rather high, especially for young clusters, this seems to be a general trend.

For the cluster cores (Fig. 10, upper panels), the trends are similar. An increase of core radii at $\left(V-M_{V}\right)>9.5$, probably comes from the definition we adopted for the cluster core.

We conclude that the apparent decrease of cluster sizes with increasing $\log t$ observed in Fig. 8 and described by Eqs. (1), (2) is generally caused by different concentration of cluster members with masses $1.3 \ldots 2.6 m_{\odot}$ with respect to the cluster centre: the concentration increases with increasing age of clusters. On the other hand, for stars of about $0.9 m_{\odot}$ the apparent distribution of linear radii does not differ considerably for young and old clusters in the Solar neighbourhood $\left(V-M_{V}<7.5\right)$. Therefore, the observed dependence of cluster sizes on age can be explained by a stronger mass segregation of stars with $m>1.3 m_{\odot}$ in old clusters rather than by a decrease of the real sizes of clusters. Of course, we cannot exclude that, with a deeper input catalogue and with a larger portion of very old clusters in a sample, a real decrease of the average cluster size with age can be found. This could be so, especially, if the location of clusters in the Galaxy is taken into account: the corresponding hints that large clusters have a lower chance to survive tidal effects are obtained in Sect. 3.

More detailed conclusions on mass segregation at $m>$ $1.3 m_{\odot}$ can be drawn if one considers the radial distribution of the most massive stars in a cluster. To illustrate this approach we show 15 clusters of different age in Fig. 11. The clusters have an extended magnitude range $\Delta V>3$ mag and are presented in a sequence of increasing age. Although the observed lower mass limit depends on the distance modulus, this is of lesser importance for the following analysis since we are interested in the upper part of the profiles (i.e., the most massive stars at a given distance from the cluster centre) only.

Guided by Fig. 11 we use the slope of the "maximum stellar mass - distance from cluster centre" relation as a statistical parameter to quantify the mass segregation effect. In order to compute this parameter, we subdivided the area of each cluster into 10 concentric rings of variable width but containing an equal number of the most probable members. This binning provides an unbiased sampling both with respect to the variation of the density profile and to the representativity of the mass distribution in a cluster. In each ring, the star with maximum mass $\log m_{\max }(r)$ was selected. Here $r$ is the distance from the cluster centre in units of the cluster radius i.e., $r=R / R_{\mathrm{cl}}, 0 \leq r \leq 1$. Of course, the most reliable results can be expected for clusters with a sufficiently large number of members covering an extended range of masses. As a compromise, we included in our analysis only clusters having a main sequence extend larger than 3 mag in the ASCC-2.5 (typically, 3.5 mag for $\log t>8$, and 6 mag for younger clusters). In order to be certain that we consider comparable mass ranges, we also required that at least one cluster member must be less massive than $2 m_{\odot}$. In total, 167 clusters meet these requirements.

For each cluster, we solved a system of linear equations describing the variations of $m_{\max }(r)$ as a function of the distance from the cluster centre

$\log m_{\max }(r)=b \times r+a$

with parameters $a$ and $b$, where $b$ describes the radial mass gradient $\mathrm{d} \log m_{\max } / \mathrm{d} r$. For illustration, the relations together with the cluster members included in the solution are shown in Fig. 11.

From the coefficients $a$ and $b$ of Eq. (3) we computed the maximum mass of a cluster member expected in the cluster centre $(r=0)$ and at the cluster edge $(r=1)$. The dependence of 


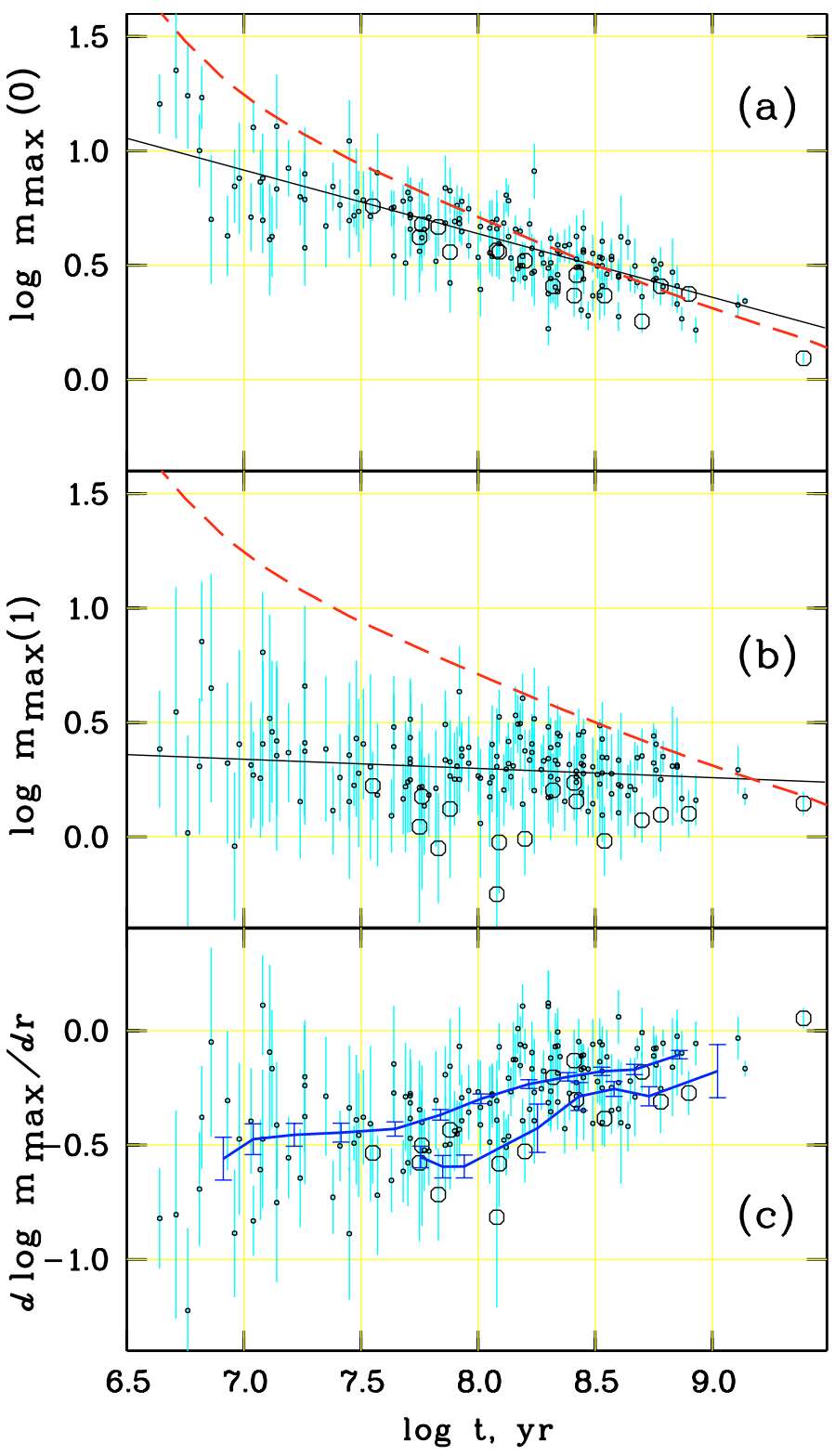

Fig. 12. The expected mass of the brightest stars computed for the cluster centres a) and edges b) versus cluster age. Panel c) shows the variation of radial mass gradient $\operatorname{dlog} m_{\max } / \mathrm{d} r$ with cluster age. Circles indicate all clusters included in the analysis, large open circles mark the subsample of nearby clusters $\left(\left(V-M_{V}\right)<7.3\right)$ with the most extended Main Sequences. The rms errors of the individual data points are shown as light grey (cyan) bars. In panels a) and b) the dashed curves show the "mass-MS-lifetime relation" based on the Padova isochrones with overshooting for $Z=0.019$, solid lines present the solution of Eqs. (4), (5). In panel $\mathbf{c}$ ) the curves show the running average of the radial mass gradient computed with a $(\log t)$-bin of 0.6 and a step of 0.2 for nearby clusters (lower curve) and for all other clusters (upper curve). The rms errors of the corresponding averages are shown with bars.

these quantities on cluster age can be well approximated by the equations

$\log m_{\max }(0)=(-0.29 \pm 0.02) \log t_{6}+(1.20 \pm 0.04)$,

$\log m_{\max }(1)=(-0.04 \pm 0.02) \log t_{6}+(0.38 \pm 0.04)$

where $t_{6}$ is the cluster age in units of Myr. The corresponding solutions are shown in Fig. 12, in panel (a) for cluster centres and in panel (b) for cluster edges.
The dashed curve in Figs. 12a,b presents a "mass-MSlifetime relation" i.e., a theoretical scale taken at the TAMS from the Padova isochrones with overshooting for $Z=0.019$. The relation sets an upper limit of masses of MS stars which can be expected in a cluster of a given age. In cluster centres, the most massive members evolve in good agreement with the "mass-MS-lifetime relation" (Fig. 12a), whereas this evolution is rather weak at the cluster edges (Fig. 12b). In absence of mass segregation Eqs. (4) and (5), which are presented by solid lines in Fig. 12a,b, should show up similar coefficients. This, however, is not the case. A large difference between masses in the central and outer regions of clusters at earlier ages indicates a strong mass segregation, on average. Due to the burning out of massive stars in the cluster cores (Fig. 12a), the mass difference is decreasing with cluster age and approaches zero at $\log t>9$. Nevertheless, one can assume that there should be members with masses lower than about $1 m_{\odot}$ in the outer regions of very old clusters, but the relatively bright limiting magnitude of the ASCC-2.5 prevents us from observing them already at $\left(V-M_{V}\right)>7$.

Variations of mass segregation with age observed in our cluster sample can be quantitatively analysed from the radial mass gradient computed by Eq. (3) and presented in Fig. 12c. A strongly negative mass gradient points out a general concentration of the most massive members to the cluster centres, whereas a large individual rms error of a mass gradient indicates mainly that relatively massive stars are present also outside the very central region (different situations are illustrated in Fig. 11).

Although, a number of clusters do not show indication for mass segregation, a systematic trend of $\mathrm{d} \log m_{\max } / \mathrm{d} r$ towards negative values can be seen over the whole range of cluster ages in Fig. 12c. At $\log t>7.6$ the radial mass gradient flattens steadily, from about -0.4 to -0.1 at $\log t \approx 8.9$. As we discussed above (Fig. 12a), this flattening can be explained by the gradual evolution of the most massive stars away from the MS in the central areas of clusters. Nearby clusters clearly support this general trend though their average curve is shifted to smaller mass gradients: in these clusters we are able to observe stars with masses slightly below $1 m_{\odot}$ which are widely distributed over the cluster area, up to the cluster edge (cf. Fig. 12b). Therefore, a stronger mass segregation can be still seen in nearby clusters with $8.5<\log t<9.0$.

The scattering of data points in Fig. 12c indicates a dependence on cluster ages. The standard deviation of the mass gradient is $0.10-0.15$ for clusters with $\log t>7.5$ and it is larger by a factor of two (0.25-0.30) for younger clusters. According to an F-test, the hypothesis of equal variances is clearly rejected for clusters younger and older than $\log t=7.5$. Also, individual rms errors of $\mathrm{d} \log m_{\max } / \mathrm{d} r$ are, on average, twice as large for younger clusters as for older ones. Taking this into account, we conclude that the group of clusters with $\log t<7.5$ is less homogeneous than the group of older clusters, and that the apparent distribution of the most massive stars over the area cannot always be described sufficiently well in young clusters by Eq. (3).

A possible explanation is that our sample at $\log t \lesssim 7.5$ presents a mixture of young clusters with a different grade of mass segregation. Some of them have a significant negative mass gradient (e.g., ASCC 58 in Fig. 11) supporting the conclusion that substantial mass segregation has occurred here already at early stages of the evolution. The absence of clusters younger than $5 \mathrm{Myr}$ in our sample prevents us, however, to understand whether the mass segregation has a primordial character (i.e. originated during cluster formation) or if it is already a result of the dynamical evolution during the first $5 \mathrm{Myr}$ of the 
cluster's life. In contrast, a few other clusters (e.g., Cr 121 in Fig. 11) have a flatter radial mass gradient, not significantly differing from zero. In these clusters, the most massive stars show a stochastic distribution over the cluster area, and it looks as if they are hampered in their dynamical evolution. Later, at about $\log t=7.7 \ldots 8.0$ the majority of clusters seems to achieve a quasiequilibrium, and the evolution of massive stars from the MS becomes a more prominent effect in the observations.

Among 31 clusters with $\log t<7.5$, our sample contains 10 clusters with significant negative mass gradients $\left(\mathrm{d} \log m_{\max } / \mathrm{d} r+3 \sigma<0\right.$ where $\sigma$ is the individual rms error of the radial mass gradient) and 6 clusters with mass gradients not significantly differing from zero $\left(\left|\mathrm{d} \log m_{\max } / \mathrm{d} r\right|<\sigma\right)$. Nevertheless, the sampling is rather poor and the scattering of data points is too large to make a statistically significant conclusion. Also, we cannot exclude the possibility that a low significance of the mass gradient can be a consequence of uncertainties in the determination of the cluster centres due to an irregular and patchy distribution of the absorbing matter within young clusters.

We conclude that the observed relation between the radial mass gradient and the age of a cluster can be interpreted in terms of the dynamical and stellar evolutions. Our sample does not include very young clusters, but at least at an age of $5 . .10$ Myr a strong radial concentration of massive stars can be already observed in several clusters. Numerical simulations (Khalisi et al. 2006) indicate that, depending on the initial mass of a cluster, mass segregation can occur very rapidly for massive members. On the other hand, this process can be hampered by stellar winds, ionisation fronts etc. of the most massive stars, especially if a cluster is located within a large star forming region (Kroupa et al. 2001). This is possibly the reason why we observe clusters of the same ages, where some show mass segregation and other do not show it at all. Nevertheless, during the following 50...100 Myr the dynamical evolution takes overhand due to pair encounters and energy equipartition, and we observe a more regular pattern in the distribution of stars of different masses. This age seems to be typical for the relaxation of clusters in our sample. Although mass segregation can still continue at lower masses, the apparent mass distribution in clusters of our sample at $\log t>8$ is mainly governed by stellar evolution removing the most massive stars from the "scene". External gravitational shocks may also influence the mass distribution in clusters and can be partly responsible for a spread of the radial mass gradient at $\log t>8$.

\section{Summary}

This study is based on the Catalogue of Open Cluster Data (COCD) and its Extension 1 described in Papers II and III. The COCD is derived from the ASCC-2.5, a homogeneous allsky catalogue with complete information on proper motions and $B, V$-photometry. So, all open clusters found in this catalogue can be treated in the same way to derive their astrophysical parameters. On the other hand, the price to be paid for this advantage is the bright completeness limit of ASCC-2.5 at about $V=11.5$. However, the biases resulting from a simply magnitude limited sample can be estimated, they have been discussed in the previous sections and have been taken care of in order not to influence the conclusions. Using samples of clusters from different sources with different photometry and/or different limiting magnitude may introduce biases in the results which cannot be estimated easily.
The whole sample from ASCC-2.5 consists of 641 open clusters. In Papers II and III we determined membership in the clusters applying photometric as well as astrometric criteria. Apparent linear radii have been computed from individual distances and angular sizes of the clusters, based on members only. For the first time, the structural properties of the galactic open cluster system have been statistically analysed from an unbiased, homogeneous, and relatively large sample. A comparison of our cluster sizes with those given in Lyngå (1987) (about 500 clusters in common) shows that cluster radii from Lyngå are in average lower by a factor of 2 , and they fit rather the core than the corona.

Our large sample allowed us to investigate the dependence of the cluster size on the age of a cluster and on its location in the Galaxy. The clusters cover an age range between about $5 \mathrm{Myr}$ to more than $1 \mathrm{Gyr}$. For younger clusters $(<200 \mathrm{Myr})$ there is no significant correlation between linear size and Galactocentric distance. At an age corresponding to two revolutions around the Galactic centre we detect that the clusters are on average smaller $\left(\overline{R_{\mathrm{cl}}}=3.8 \pm 0.2 \mathrm{pc}\right)$ inside the solar circle than outside $\left(\overline{R_{\mathrm{cl}}}=4.6 \pm 0.3 \mathrm{pc}\right)$. According to a $(K-S)$ test the probability that both subsamples are drawn from the same distribution is less than $4 \%$. This size dependence on Galactocentric radius lead to the conclusion that the inner Galactic disk is void with respect to older open clusters. No clusters older than the age of the Hyades should exist inside a Galactocentric radius of about $6 \mathrm{kpc}$. Perpendicular to the plane we note a systematic increase of cluster sizes with increasing $\left|Z^{\prime}\right|$. This, however, turned out to be significant only for clusters older than $\log t>8.35$, which already survived at least one revolution around the Galactic centre.

From these findings the following picture of the evolution of open clusters arises. Clusters in the wider Solar neighbourhood are formed within the thin disk, their initial size distribution does not show a significant correlation with the $R_{\mathrm{G}^{-}}$and $\left|Z^{\prime}\right|$ - coordinates. The size distribution changes at ages corresponding to one revolution around the Galactic centre. At low $Z^{\prime}$ we now note a relatively larger number of small clusters. This makes us conclude that close to the Galactic equator and inside the solar circle larger clusters are in danger to dissolve even during the first revolution around the Galactic centre. On the other hand, they have a higher chance to survive encounters and the impact of Galactic tidal forces, if their orbits are outside the Solar one and are inclined to the Galactic plane. Therefore, they reach higher ages at these locations. Finally, the apparent linear sizes of clusters and their cores are, on average, decreasing with time and this process is faster for the coronae than for the cores. Taking into account that our input catalogue is magnitude limited, this finding can be interpreted as a first hint for mass segregation.

In the majority of clusters of our sample clear evidence for mass segregation of stars with $m>1 m_{\odot}$ has been established from the distribution of the radial mass gradient as a function of age. An apparent flattening of the radial mass gradient for clusters older than 50...100 Myr occurs due to stellar evolution when massive stars subsequently leave the main sequence, and, secondly, because we cannot observe the low-mass stars due to the bright limiting magnitude of the ASCC-2.5. External gravitational shocks may also influence the mass distribution in clusters and can be partly responsible for a spread of the radial mass gradient at $\log t>8$. Nevertheless, a "typical" cluster older than about 100 Myr and within about $1 \mathrm{kpc}$ from the Sun shows mass segregation.

The youngest clusters of our sample with ages less than 50 Myr show a large spread of the radial mass gradient: from 
clusters with a clear concentration of the most massive stars to the centres up to clusters with no or only a flat mass gradient. The different dynamical state of clusters of the same age possibly results from the different initial conditions and environments of the clusters.

Acknowledgements. This work was supported by the DFG grant 436 RUS 113/757/0-1, and RFBR grant 06-02-16379. We acknowledge a discussion of the results with Rainer Spurzem. We are grateful to the anonymous referee for his/her useful comments.

\section{References}

Adams, J. D., Stauffer, J. R., Monet, D. G., Skrutskie, M. F., \& Beichman, C. A. 2001, AJ, 121, 2053

Adams, J. D., Stauffer, J. R., Skrutskie, M. F., et al. 2002, AJ, 124, 1570

Andersen, J., \& Nordström, B. 2000, in Stellar Clusters and Associations, ed. R. Pallavicini, G. Micela, \& S. Scioortino, ASP Conf. Proc., 198, 171

Becker, W., \& Fenkart, R. 1971, A\&AS, 4, 241

Bergond, G., Leon, S., \& Guibert, J. 2001, A\&A, 377, 462

Bonatto, C., \& Bica 2005, A\&A, 437, 483

Bonnell, I. A., \& Davies, M. D. 1998, MNRAS, 295, 691

Burki, G., \& Maeder, A. 1976, A\&A, 51, 247

Danilov, V. M., \& Seleznev, A. F. 1993, Astron. Astrophys. Trans., 6, 293

Fischer, P., Pryor, C., Murray, S., Mateo, M., \& Richtler, T. 1998, AJ, 115, 952

Friel, E. D. 1995, ARA\&A, 33, 381

de Grijs, R., Johnson, R. A., Gilmore, G. F., \& Frayn, C. M. 2002a, MNRAS, 331,228

de Grijs, R., Gilmore, G. F., Johnson, R. A., \& Mackey, A. D. 2002b, MNRAS, 331,245

de Grijs, R., Gilmore, G. F., Mackey, A. D., et al. 2002c, MNRAS, 337, 597

Girardi, L., Bertelli, G., Bressan, A., et al. 2002, A\&A, 391, 195
Hillenbrand, L. A. 1997, AJ, 113, 1733

Hillenbrand, L. A., \& Hartmann, L. W. 1998, ApJ, 492, 540

Høg, E., Fabricius, C., Makarov, V. V., et al. 2000, A\&A, 355, L27

Janes, K. A., Tilley, C., \& Lyngå, G. 1988, AJ, 95, 771

Jones, B. F., \& Stauffer, J. R. 1991, AJ, 102, 1080

Khalisi, E., Amaro-Seoane, P., \& Spurzem, R. 2006, MNRAS, submitted [arXiv:astro-ph/0602570]

Kharchenko, N. V. 2001, Kinematics and Physics of Celestial Bodies, 17, 409

Kharchenko, N. V., Pakulyak, L. K., \& Piskunov, A. E. 2003, Astron. Rep., 47, 263

Kharchenko, N. V., Piskunov, A. E., Röser, S., Schilbach, E., \& Scholz, R.-D. 2004, Astron. Nachr., 325, 743 (Paper I)

Kharchenko, N. V., Piskunov, A. E., Röser, S., Schilbach, E., \& Scholz, R.-D. 2005a, A\&A, 438, 1163 (Paper II)

Kharchenko, N. V., Piskunov, A. E., Röser, S., Schilbach, E., \& Scholz, R.-D. 2005b, A\&A, 440, 403 (Paper III)

Kroupa, P., Aarseth, S., \& Hurley, J. 2001, MNRAS, 321, 699

Lyngå, G. 1982, A\&A, 109, 213

Lyngå, G. 1987, Catalogue of open clusters data, Fifth edition (Strasbourg: CDS, VII/92)

Mathieu, R. D. 1984, ApJ, 284, 643

Nilakshi, Sagar, R., Pandey, A. K., \& Mohan, V. 2002, A\&A, 383, 153

Piskunov, A. E., Kharchenko, N. V., Röser, S., Schilbach, E., \& Scholz, R.-D. 2006, A\&A, 445, 545 (Paper IV)

Portegies Zwart, S. F., \& McMillan, S. L. W. 2002, ApJ, 576, 899

Raboud, D., \& Mermilliod, J.-C. 1998a, A\&A, 329, 101

Raboud, D., \& Mermilliod, J.-C. 1998b, A\&A, 333, 897

Sagar, R., Myakutin, V. I., Piskunov, A. E., \& Dluzhnevskaya, O. B. 1988, MNRAS, 234, 831

Slesnick, C. L., Hillenbrand, L. A., \& Massey, P. 2002, ApJ, 576, 880

Spitzer, L., \& Chevalier, R. A. 1973, ApJ, 183, 565

Spitzer, L., \& Shull, J. M. 1975, ApJ, 201, 773

Tadross, A. L., Werner, P., Osman, A., \& Marie, M. 2002, NewA, 7, 553 